\title{
Nemo-like kinase is critical for p53 stabilization and function in response to DNA damage
}

\author{
H-H Zhang ${ }^{1,3}$, S-Z Li ${ }^{1,3}$, Z-Y Zhang ${ }^{1}$, X-M Hu${ }^{1}$, P-N Hou', L Gao ${ }^{2}$, R-L Du*,1 and X-D Zhang, ${ }^{*, 1}$
}

The DNA damage response (DDR) acts as a protective mechanism for maintaining cell homeostasis. Nemo-like kinase (NLK) is a serine/threonine-protein kinase that has an important role in many pathways; however, its function in the DDR has not yet been defined. In our study, NLK-deficient HCT116 cells were found to be resistant to etoposide-induced cell death. We demonstrated that NLK is required for p53 activation in response to DNA damage. Remarkably, mechanistic studies revealed that NLK interacts with p53 and stabilizes p53 by blocking MDM2-mediated p53 ubiquitination and degradation. Furthermore, NLK enhances p53 activity and affects expression downstream of p53. Interestingly, these functions of NLK are not related to its kinase activity. Consistent with these results, NLK-deficient cells have a resistance effect on DNA damage. Therefore, these findings emphasize that NLK is a novel factor in DDR mechanisms.

Cell Death and Differentiation (2014) 21, 1656-1663; doi:10.1038/cdd.2014.78; published online 13 June 2014

Cells are constantly in danger, threatened by exogenous and endogenous factors; each cell has a set of efficient and complex DNA damage response (DDR) mechanisms to maintain homeostasis. ${ }^{1,2}$ DDR mechanisms involve many factors, particularly $\mathrm{p} 53 .^{2}$

p53 is a transcription factor, and it is one of the most important tumor suppressors in human cancer. Wild-type p53 is a guardian of the genome ${ }^{3}$ because it is activated in response to DNA damage. ${ }^{4,5}$ p53 has an important role in cell cycle arrest, DNA repair and apoptosis in response to genotoxic and cellular stress. ${ }^{2,6}$ Mutations of the p53 gene lead to a high risk of cancer, and cells lacking functional p53 are functionally deficient. Under normal conditions, the protein level of p53 remains low because of MDM2-mediated ubiquitination and degradation. ${ }^{7,8}$ In stressful conditions, posttranslational modifications such as phosphorylation, acetylation and ubiquitination regulate p53 stability and activity. ${ }^{8,9}$ There are also some transcriptional coactivators or corepressors that modulate the activity of P53.,10 However, it has still not yet been determined how p53 activity is affected by stressful conditions.

Nemo-like kinase (NLK), a member of the mitogenactivated protein kinase family, is a serine/threonine-protein kinase that has roles in various developmental events, ${ }^{11-13}$ particularly neural development and maintenance. ${ }^{14}$ It has been reported that NLK is a positive effector of the noncanonical Wnt signaling pathway ${ }^{14}$ and that NLK suppresses a wide range of transcription factors, including NF- $\kappa$ B. ${ }^{11,15}$ Some studies have shown that NLK is involved in apoptosis induction in DLD-1 human colon and prostate cancer cells. ${ }^{14,16,17}$ However, the function of NLK in repairing DNA damage is not certain. Because p53 has such an important role in DNA repair, we tested whether NLK influences the ability of p53 to escape degradation. Here, our results indicate that DNA damage induces NLK and that this induction is related to $\mathrm{p53}$ variation. Furthermore, we showed that NLK physically interacts with p53 using coimmunoprecipitation experiments. Reporter assays and ubiquitination detection results demonstrated that NLK can stabilize p53 protein levels and enhance p53 activity by inhibiting MDM2-mediated p53 ubiquitination. These results suggest that NLK is a novel regulator of $\mathrm{p} 53$.

\section{Results}

NLK-deficient cells are resistant to DNA damage. The integrity of the DNA is challenged by a variety of agents, including environmental agents and a wide variety of drugs used in chemotherapy. ${ }^{18}$ Etoposide (Eto) is an anticancer drug that functions as a topoisomerase inhibitor. ${ }^{19}$ Eto causes errors in DNA synthesis and promotes apoptosis in cancer cells. ${ }^{20}$ Remarkably, when we treated cells with Eto, we found that NLK-deficient HCT116 cells showed increased resistance to DNA damage when compared with wild-type HCT116 cells (Figure 1a).

NLK is required for p53 activation in response to DNA damage. To investigate whether NLK is involved in the genotoxic stress response, we treated human colorectal carcinoma cells from the HCT116 (wild-type p53) cell line with various agents. NLK production was induced by these agents, particularly Eto and doxorubicin (Figure 1b). We observed that Eto induction increases NLK protein levels concomitant with p53 protein induction (Figure 1c).

\footnotetext{
${ }^{1}$ Department of Cell Biology, College of Life Sciences, Wuhan University, Wuhan, China and ${ }^{2}$ Department of Cardiology, Institute of Cardiovascular Disease, Union Hospital, Tongji Medical College, Hua Zhong University of Science and Technology, Wuhan, China

*Corresponding authors: R-L Du or X-D Zhang, Department of Cell Biology, College of Life Sciences, Wuhan University, Wuhan 430072, China. Tel/Fax: + 862768752099 ; E-mail: runleidu@ whu.edu.cn (R-LD) or Tel/Fax: + 8627 68756606; E-mail: zhangxd@whu.edu.cn (X-DZ)

${ }^{3}$ These authors contributed equally to this work.

Abbreviations: NLK, Nemo-like kinase; DDR, DNA damage response; Eto, Etoposide; FBS, fetal bovine serum

Received 27.1.14; revised 30.4.14; accepted 05.5.14; Edited by M Oren; published online 13.6.14
} 
a

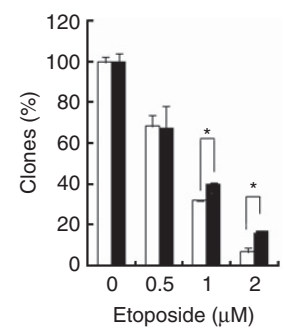

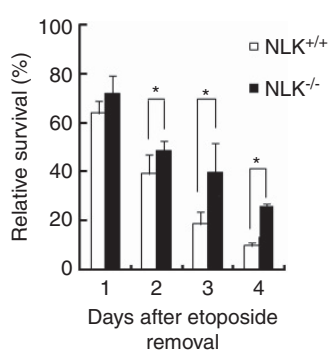

$\square \mathrm{NLK}^{+/+}$

$\mathrm{NLK}^{-1-}$

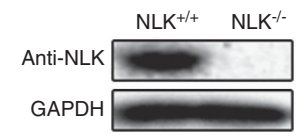

c

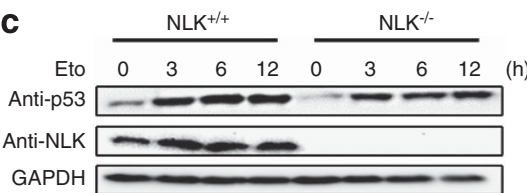

GAPDH

b

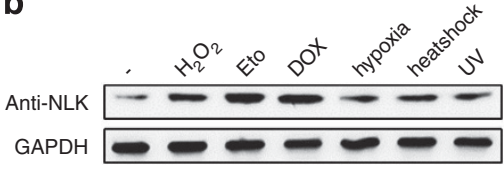

(h)

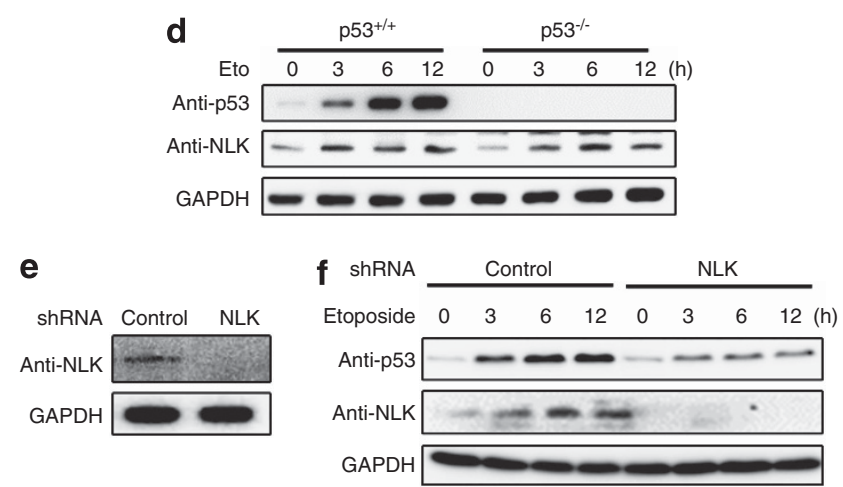

Figure 1 NLK-deficient cells show DNA damage resistance, and NLK is required for the activation of p53 in response to DNA damage. (a) (Left) Clone formation. HCT116 $\mathrm{NLK}^{+1+}$ cells and HCT116 NLK ${ }^{-1-}$ cells $\left(2 \times 10^{3}\right.$ cells each) were seeded in six-well plates and treated the following day with or without Eto as indicated; after $24 \mathrm{~h}$, the medium was replaced with fresh McCoy's $5 \mathrm{~A}$ medium. Ten days later, the clones were stained with crystal violet, and clone formation was analyzed. (Middle) Cell survival analysis. Cells $\left(1 \times 10^{4}\right)$ were seeded in a 96-well plate and treated the following day with $0.5 \mathrm{mM}$ Eto; after $24 \mathrm{~h}$, the medium was replaced with fresh McCoy's $5 \mathrm{~A}$ medium, and cell survival analysis was performed using a CCK8 kit. (Right) Immunoblot analysis verified the NLK expression levels in both wild-type and NLK knockout HCT116 cells. ${ }^{*} P<0.05$. (b) HCT116 cells were treated with $\mathrm{H}_{2} \mathrm{O}_{2}(0.5 \mathrm{mM}, 6 \mathrm{~h})$, Eto $(0.5 \mathrm{mM}, 6 \mathrm{~h})$, doxorubicin $(1 \mu \mathrm{g} / \mathrm{ml}, 6 \mathrm{~h})$, hypoxia $(1 \mathrm{~h})$, heat shock $\left(43^{\circ} \mathrm{C}, 1 \mathrm{~h}\right)$ or UV irradiation $\left(50 \mathrm{~J} / \mathrm{m}^{2}\right)$. Cells were then harvested, and the NLK protein levels were determined using immunoblot analysis. (c) HCT116 NLK ${ }^{+1+}$ and HCT116 NLK ${ }^{-1-}$ cells were treated with $0.5 \mathrm{mM}$ Eto and were harvested at the indicated times. Immunoblot analysis was performed using NLK- and p53-specific antibodies. (d) $\mathrm{HCT}_{116} \mathrm{p} 53^{+/+}$and HCT116 p53 $3^{-I-}$ cells were treated with $0.5 \mathrm{mM}$ Eto, and cells were harvested at the indicated times. Immunoblot analysis was performed using NLK and p53-specific antibodies. (e) HCT116 cells were infected with a lentivirus expressing an shRNA specific for NLK (NLK-shRNA) or a control shRNA (shRNA-control). Seventy-two hours post infection, immunoblot analysis was performed to detect NLK protein expression levels. Protein-specific bands were normalized to GAPDH. (f) HCT116 cells were infected with a lentivirus expressing NLK-shRNA or shRNA-control, and the cells were treated with $0.5 \mathrm{mM}$ Eto and harvested at the indicated times. Immunoblot analysis was performed using NLK- and p53specific antibodies

To explore whether NLK induction depends on p53 activation, we examined the NLK level in colorectal carcinoma HCT116 $553^{+/+}$and HCT116 $553^{-1-}$ cells. We found similar increases in NLK protein in both cell types, as presented in Figure 1d. These results indicate that NLK induction in response to DNA damage is p53 independent.

To determine whether NLK contributes to p53 induction in response to DNA damage, we used NLK-deficient HCT116 cells (under review in BBA Molecular Cell Research) that were constructed using somatic cell knockout technology ${ }^{21,22}$ (Figure 1a right); we then treated these knockout cells with Eto. The resultant increase in p53 levels was dramatically lower in the NLK-deficient cells compared with NLK-wild type cells (Figure 1c). To confirm this result, we also performed NLK knockdown experiments to investigate the changes in the p53 levels. HCT116 cells were infected with a lentivirus expressing an shRNA specific for NLK (NLK-shRNA) or a control shRNA (control-shRNA). The NLK-shRNA knockdown efficiency was measured by immunoblot analysis (Figure 1e). The infected cells were treated with Eto, and the resultant increase in p53 levels was also dramatically lower in the NLK-shRNA cells compared with control-shRNA cells (Figure 1f). These results demonstrate that NLK is required for the upregulation of p53 expression in response to DNA damage.

NLK regulates $\mathrm{p53}$ protein stability and activity. To elucidate the above results, we examined whether NLK regulates p53 protein stability. As shown in Figure 2a, p53 expression was elevated when NLK was overexpressed in HCT116 $553^{-1-}$ cells, and this response occurred in a dosedependent manner. Surprisingly, as shown in Figure 2b, NLK kinase activity had no effect on the stability of p53, as expressing the NLK kinase mutation vector NLK155M also increased the levels of p53 expression. The results of a reporter assay using a p53 response reporter in HCT116

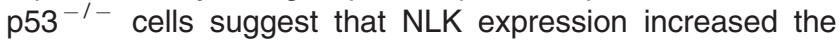


p53-mediated transcription activity (Figure 2c), and the results in Figure $2 d$ indicate that this activation occurred in a dose-dependent manner. Similarly, NLK155M expression also increased p53-mediated transcription activity (Figure 2e). p53 activity decreased in NLK-deficient cells (Figure 2f) that were treated with Eto. We confirmed this result in the shRNA cells and demonstrated that p53 activity decreased in NLK-shRNA cells treated with Eto (Figure 2g). Taken together, these results demonstrate that NLK regulates p53 stability and increases its transcriptional activity, and these effects are not associated with NLK kinase activity.

NLK interacts with p53. It has been reported that NLK is predominately localized in the nucleus, ${ }^{11}$ and, interestingly,
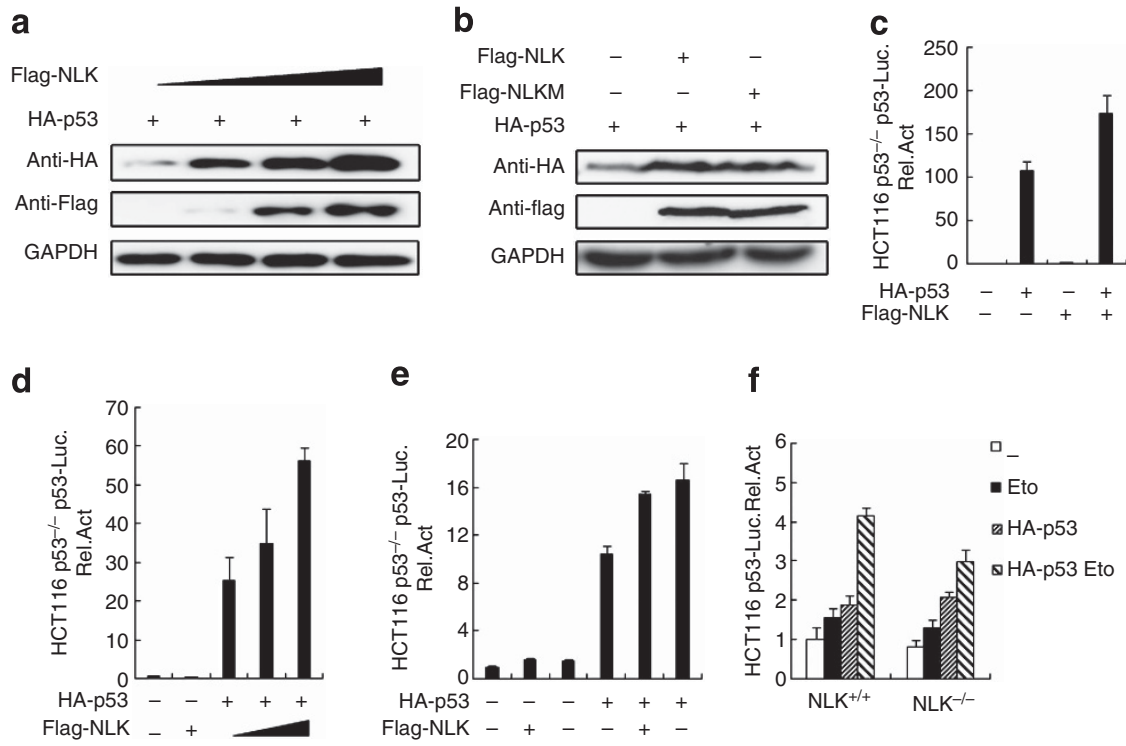

e

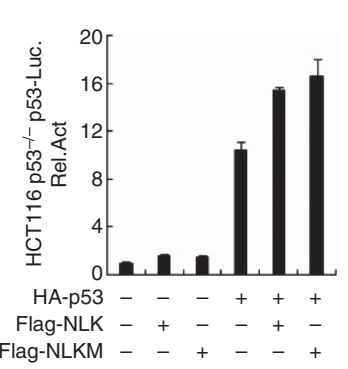

f
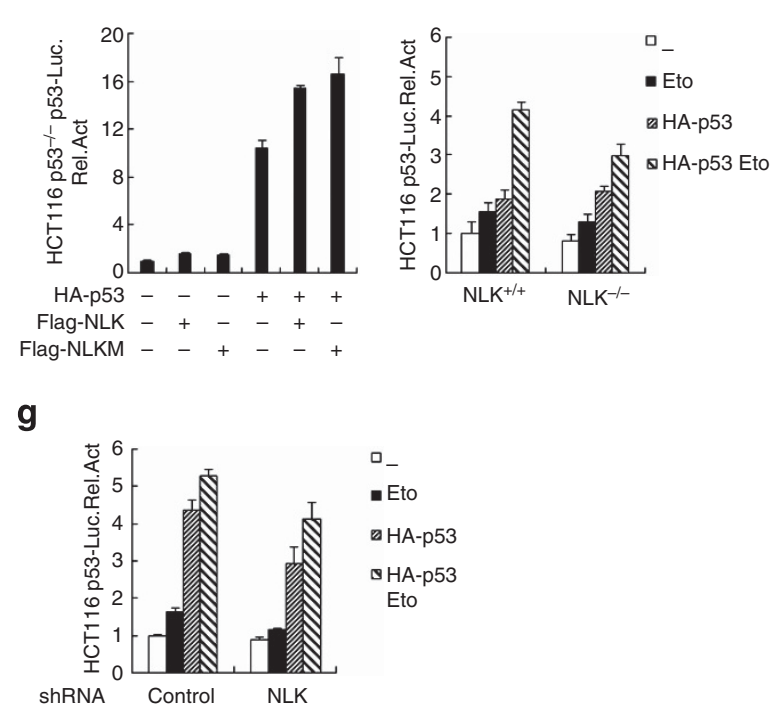

Figure 2 NLK regulates p53 protein stability and activity. (a and b) NLK regulates p53 protein stability. (a) HCT116 p53-1- cells were transfected with $0.5 \mu \mathrm{g}$ of HA-p53 or with increasing amounts of Flag-NLK plasmids $(0.5,1$ and $2 \mu \mathrm{g})$. (b) Cells were transfected with or without $0.5 \mu \mathrm{g}$ of Flag-NLK or Flag-NLKM plasmids. Cell lysates were analyzed by immunoblot analysis with Flag- and HA-specific antibodies. (c-e) NLK regulates p53 activity. HCT116 p53-1- cells were co-transfected with $0.5 \mu \mathrm{g}$ of the p53-responsive reporter pp53-TA-luc and $100 \mathrm{ng}$ of HA-p53. (c) Cells were transfected with $100 \mathrm{ng}$ of the Flag-NLK plasmids. (d) Cells were transfected with increasing amounts of the Flag-NLK plasmids. (e) Cells were transfected with $100 \mathrm{ng}$ of Flag-NLK or $100 \mathrm{ng}$ of Flag-NLKM plasmids, as indicated. Luciferase activity was measured $24 \mathrm{~h}$ later. (f and $\mathbf{g}$ ) NLK is required for p53 activity in response to DNA damage. (f) HCT116 NLK ${ }^{+1+}$ and HCT116 NLK ${ }^{-1-}$ cells were co-transfected with $0.5 \mu \mathrm{g}$ of the p53-responsive reporter pp53-TA-luc, with or without $100 \mathrm{ng}$ of HA-p53, as indicated. The cells were treated with or without $0.5 \mathrm{mM}$ Eto as indicated. The luciferase activity was measured $6 \mathrm{~h}$ after Eto treatment. $(\mathbf{g}) \mathrm{HCT} 116$ cells were infected with NLK-shRNA or control-shRNA lentivirus, and the cells were then co-transfected with $0.5 \mu \mathrm{g}$ of the p53-responsive reporter pp53-TA-luc, with or without $100 \mathrm{ng}$ of HA-p53, as indicated. The cells were treated with or without $0.5 \mathrm{mM}$ Eto, as indicated. Twenty-four hours later, the luciferase activity was measured

\footnotetext{
Figure 3 NLK interacts with p53. (a) Colocalization of GFP-p53 and cherry-NLK in the nuclei of HCT116 cells. HCT116 cells were co-transfected with $1 \mu \mathrm{g}$ of GFP-p53 and $1 \mu \mathrm{g}$ of cherry-NLK. Twenty-four hours after transfection, the cells were treated with or without $0.5 \mathrm{mM}$ Eto for $10 \mathrm{~h}$; then, the cells were fixed with $4 \%$ paraformaldehyde, and images were captured using an Olympus Laser Scanning Confocal Microscope. (b) HEK293 cells were transfected with HA-p53 and Flag-NLK expression vectors as indicated. Twenty-four hours later, the cell lysates were immunoprecipitated with anti-Flag M2 affinity gel and analyzed by immunoblot analysis with the indicated antibodies. (c) HCT116 lysates were immunoprecipitated with an anti-p53 (DO-1) antibody, and normal lgG was used as a negative control. Immunoblot analysis was performed using NLK- and p53-specific antibodies. (d) HCT116 cells and HCT116 cells stably expressing Flag-NLK were treated with or without $0.5 \mathrm{mM}$ Eto, as indicated, for $12 \mathrm{~h}$; the cell lysates were then immunoprecipitated using an anti-p53 (DO-1) antibody, and normal IgG was used as a negative control. Immunoblot analysis was performed using Flag- and p53-specific antibodies. (e) GST pull-down assays were performed with the indicated GST-fused proteins and cell lysates from HCT116 cells stably expressing Flag-NLK and were analyzed by immunoblot analysis with the indicated antibodies. (f) GST-NLK and His-p53 proteins were expressed in bacteria and purified. GST pull-down assays were performed with the indicated GST- and His-fused proteins and were analyzed by immunoblot analysis with the indicated antibodies. (g) HEK293 cells were co-transfected with HA-p53 or its truncations and Flag-NLK vectors. Twenty-four hours later, cell lysates were immunoprecipitated with anti-Flag M2 affinity gel and analyzed by immunoblot analysis with the indicated antibodies. (h) HEK293 cells were co-transfected with HA-p53 and Flag-NLK or its truncation vectors. Twenty-four hours later, cell lysates were immunoprecipitated with anti-Flag M2 affinity gel and analyzed by immunoblot analysis with the indicated antibodies
} 
p53 is also mainly localized in the nucleus. Confocal laser scanning microscopy showed that p53 and NLK colocalize within the nucleus, especially in cells treated with Eto (Figure 3a), raising the possibility that NLK and p53 directly interact. To explore this possibility, we performed a coimmunoprecipitation experiment, as shown in Figure $3 b^{5}$

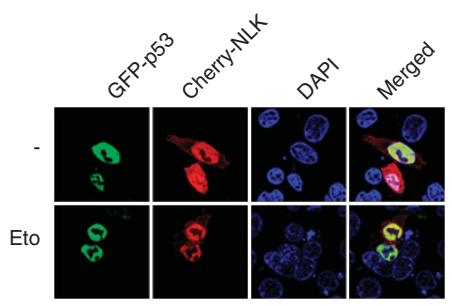

C

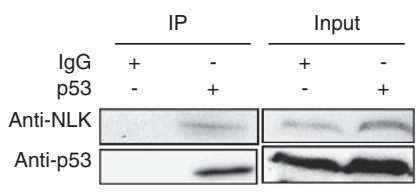

e

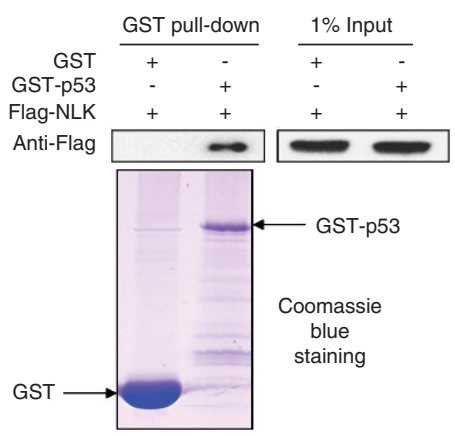

$\mathbf{g}$

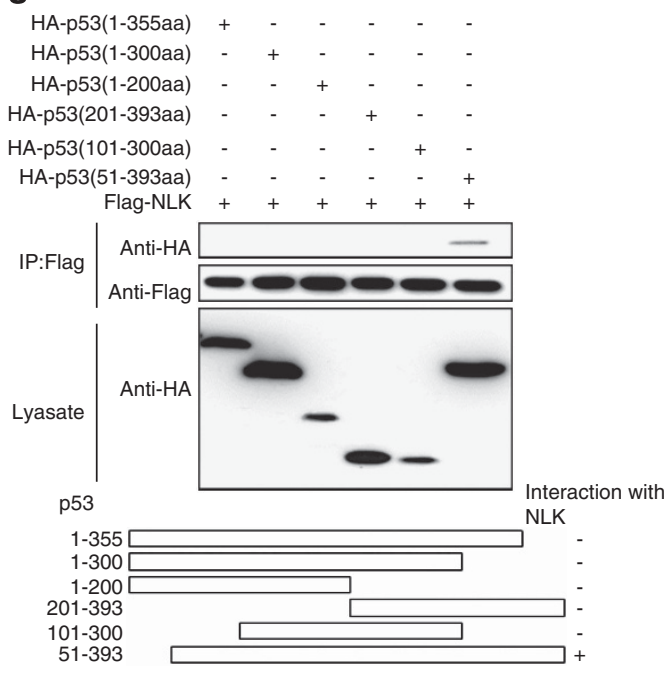

Flag-NLK and HA-p53 expression vectors were separately transfected or co-transfected into HEK293 cells. We investigated the potential interactions between Flag-NLK and HAp53. We also examined the in vivo interaction of endogenous p53 and NLK in HCT116 cells using an anti-p53 antibody (Figure $3 \mathrm{c}$ ). The interaction between p53 and NLK was

b

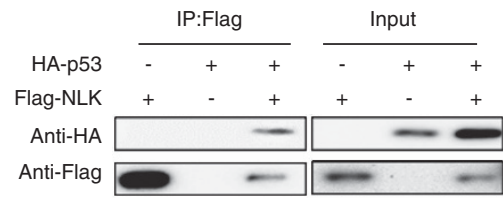

d

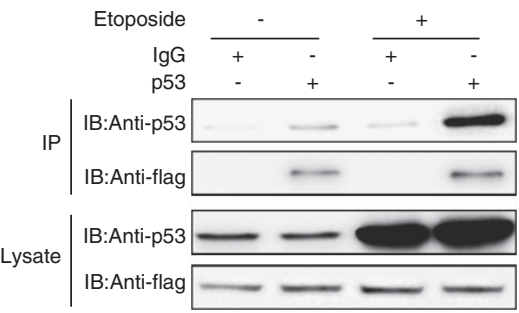

f

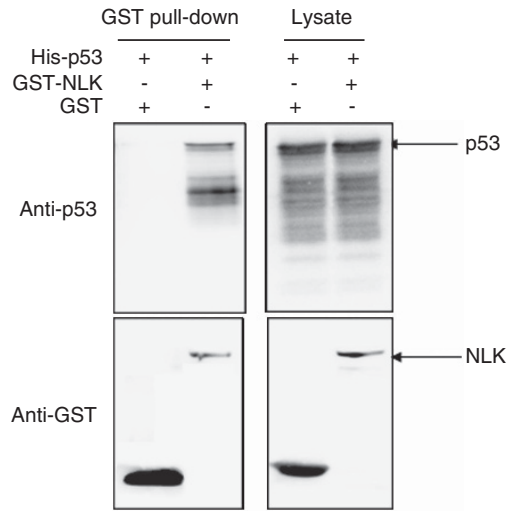

h

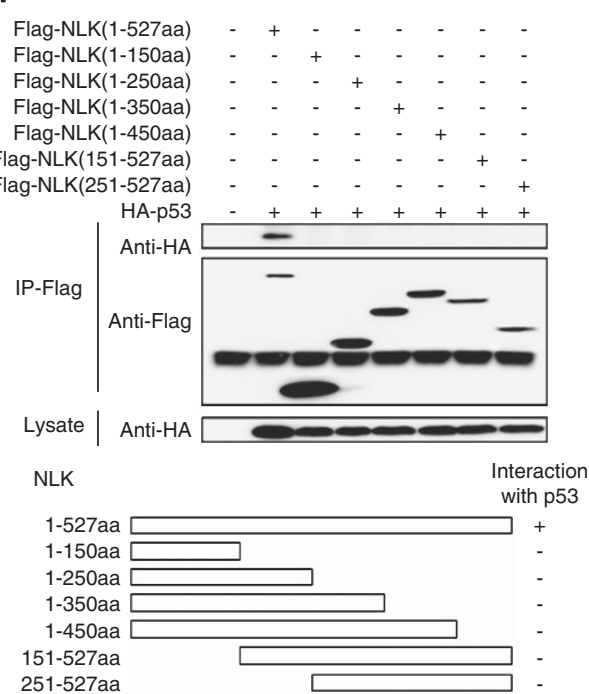


intensified when cells were treated with Eto (Figure 3d). This interaction was further confirmed using a GST pull-down assay. GST-p53 specifically interacted with transfected Flag$\mathrm{NLK}$, as shown in Figure $3 e$. To determine whether the interaction between p53 and NLK is direct, GST-NLK and His-p53 were expressed in bacteria and purified. Their interaction was confirmed using a GST pull-down assay (Figure 3f). As expected, we found that NLK and p53 could directly interact. These data demonstrate that NLK directly interacts with p53.

Next, we examined the binding domain preference between p53 and NLK. We transfected HA-p53 mutant and Flag-NLK expression vectors into HEK293 cells and performed coimmunoprecipitation experiments using anti-Flag M2 affinity gel (Figure 3g). Interaction was only detected with the 51-393 p53 mutant. We also performed co-immunoprecipitation experiments using Flag-NLK mutants and HA-p53, and as shown in Figure 3h, HA-p53 only interacts with full-length NLK. We presume, therefore, that the structural integrities of p53 and NLK are important for their interaction.

NLK affects p53 ubiquitination. The ubiquitin-proteasome pathway is the main degradation pathway of p53; if p53 were stabilized by NLK, we would expect to see NLK affect p53 degradation. As shown in Figure $4 \mathrm{a}$, NLK overexpression resulted in decreased p53 ubiquitination. MDM2 is a major regulator of ubiquitin-mediated p53 degradation; therefore, we chose to investigate whether NLK affects MDM2-mediated p53 degradation. As shown in Figures 4b, p53 protein levels were reduced when HA-p53 and Myc-MDM2 were co-transfected into HCT116 cells. However, the protein levels were clearly restored once Flag-NLK was co-transfected. Reporter assay results suggest that MDM2 overexpression decreased p53 activity and that NLK overexpression eliminated this effect (Figure $4 \mathrm{c}$ ). The results of the reporter assay also showed that this elimination of overexpression was not associated with NLK kinase activity (Figure 4c). Therefore, these results demonstrate that NLK blocks MDM2-mediated p53 ubiquitination and degradation.

Next, we investigated whether NLK disrupts the p53MDM2 interaction. When NLK was overexpressed, coprecipitation of p53 with MDM2 was dramatically decreased (Figure 4d), suggesting that NLK disrupts the interaction between p53 and MDM2. We also examined the in vivo interaction between endogenous p53 and MDM2 in HCT116 cells and HCT116 $\mathrm{NLK}^{-1-}$ cells using an anti-p53 antibody, and the results suggest that NLK deficiency may enhance the interaction between p53 and MDM2 (Figure 4e). We performed co-immunoprecipitation experiments, as shown in Figure 4f, and detected an interaction between Flag-NLK and Myc-MDM2. Therefore, NLK interferes with the interaction between MDM2 and p53 and, as a result, inhibits MDM2mediated p53 ubiquitination and degradation.

NLK affects p53 acetylation and downstream gene expression. It has been reported that acetylation of p53
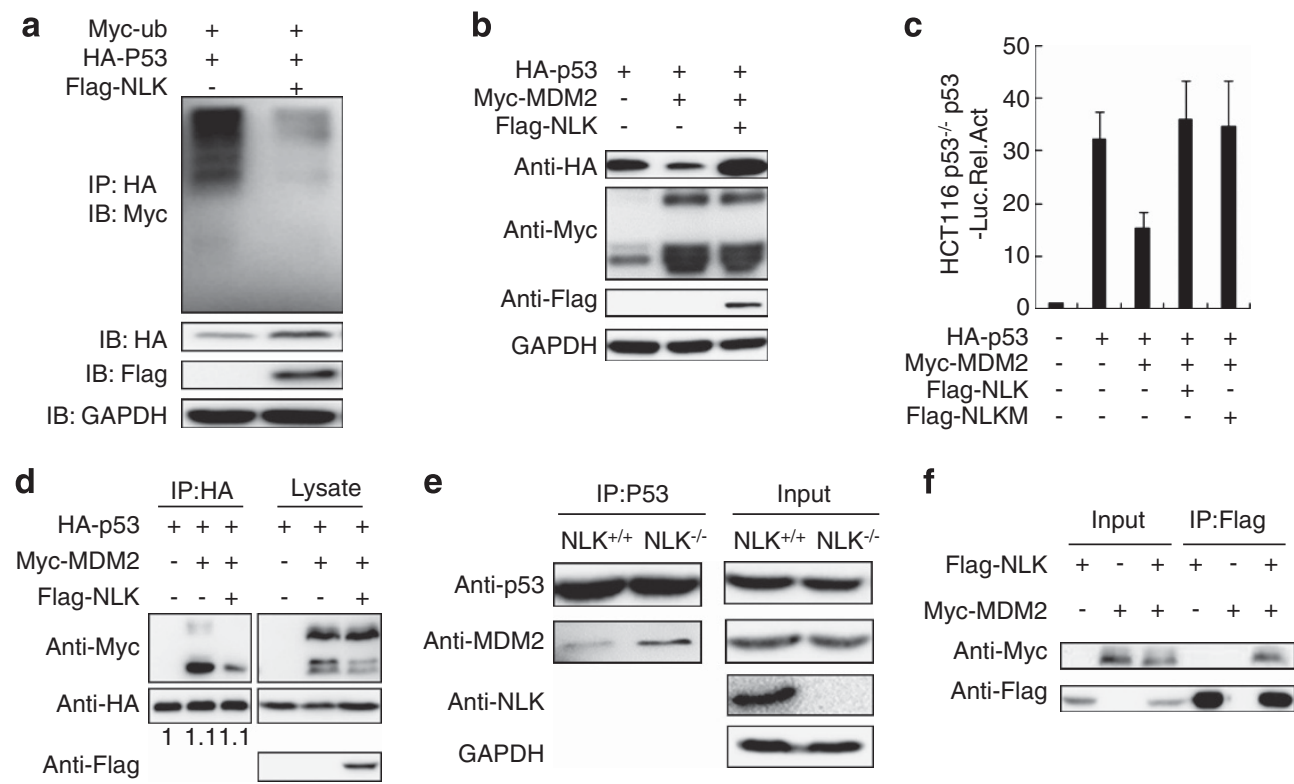

Figure 4 NLK affects p53 ubiquitination. (a) NLK blocks p53 ubiquitination. HCT116 cells were transfected with $1 \mu \mathrm{g}$ of Myc-ubiquitin-, $1 \mu \mathrm{g}$ of HA-p53- and $1 \mu \mathrm{g}$ of FlagNLK-expressing plasmids. Thirty-six hours after transfection, the cell lysates were immunoprecipitated with anti-Flag M2 affinity gel and analyzed by immunoblot analysis with the indicated antibodies. (b) NLK blocks MDM2-mediated p53 degradation. HEK293 cells were transfected with $1 \mu \mathrm{g}$ of HA-p53, $1 \mu \mathrm{g}$ of Myc-MDM2 and $1 \mu \mathrm{g}$ of Flag-NLK

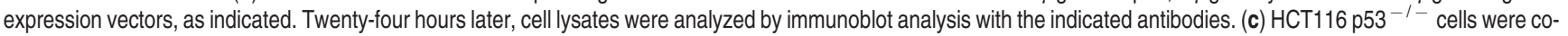
transfected with $0.5 \mu \mathrm{g}$ of the p53-responsive reporter pp53-TA-luc, $100 \mathrm{ng}$ of HA-p53, $100 \mathrm{ng}$ of Myc-MDM2, $100 \mathrm{ng}$ of Flag-NLK and $100 \mathrm{ng}$ of Flag-NLKM plasmids, as indicated. Luciferase activity was measured $24 \mathrm{~h}$ after transfection. (d) NLK disrupts the p53-MDM2 interaction. HEK293 cells were transfected with $1 \mu \mathrm{g}$ of $\mathrm{HA}-\mathrm{p} 53-, 1 \mu \mathrm{g}$ of Myc-MDM2 and $1 \mu \mathrm{g}$ of Flag-NLK expression vectors, as indicated. Twenty-four hours later, cell lysates were immunoprecipitated with anti-Flag M2 affinity gel and analyzed by immunoblot analysis with the indicated antibodies. (e) NLK disrupts the endogenous p53-MDM2 interaction. HCT116 NLK ${ }^{+I+}$ and HCT116 NLK ${ }^{-I-}$ cell lysates were immunoprecipitated with an anti-p53 (DO-1) antibody and analyzed by immunoblot analysis using the indicated antibodies. (f) NLK interacts with MDM2. HEK293 cells were transfected with $1 \mu \mathrm{g}$ of Myc-MDM2 and $1 \mu \mathrm{g}$ of Flag-NLK expression vectors, as indicated. Twenty-four hours later, cell lysates were immunoprecipitated with anti-Flag M2 affinity gel and analyzed by immunoblot analysis using the indicated antibodies 
promotes p53 stabilization and activation, ${ }^{9,23}$ and competition between ubiquitination and acetylation affects p53 stability. We next investigated whether NLK affected p53 acetylation. As expected, acetylation of p53 at Lys382 was decreased in the Eto-treated HCT116 $\mathrm{NLK}^{-/-}$cells (Figure 5a). Further, we found that the expression of NLK restored p53 acetylation at Lys382 in the presence of MDM2 (Figure $5 b$ ). Therefore, NLK may stabilize p53 by enhancing p53 acetylation.

p53 is a transcription factor that may bind to promoters of its specific target genes in response to DNA damage. ${ }^{24}$ Because our results suggest that NLK regulates p53 activity, we investigated whether NLK is required for p53-targeted gene activity. We examined the expression of $\mathrm{p} 21$, a target gene of p53, in HCT116 $\mathrm{NLK}^{+/+}$and HCT116 $\mathrm{NLK}^{-1-}$ cells treated with Eto (Figure $5 \mathrm{c}$ ). As expected, the $\mathrm{p} 21$ protein levels were lower in the HCT116 $\mathrm{NLK}^{-1-}$ cells, which is consistent with the p53 protein levels. We also tested the p21 protein levels in the HCT116 p53 ${ }^{+/+}$and HCT116 p53 ${ }^{-I-}$ cells (Figure $5 \mathrm{~d}$ ). These results suggest that NLK is required for $p 53$ to activate p21 expression. Thus, NLK may regulate p53 function and activity.

\section{Discussion}

We have identified NLK as a novel factor involved in the cellular response to DNA damage, and our results suggest that NLK is crucial for p53 activation in response to DNA damage. Under normal conditions, p53 is regulated by MDM2, which is an E3 ligase involved in p53 ubiquitination and degradation. ${ }^{7,8}$ In response to DNA damage, p53 is a pivotal factor that is rapidly accumulated and activated following ATM/ATR kinase activation. ${ }^{8,25}$ Next, post-translational modification factors modify p53 to maintain its stability and activity. ${ }^{8}$ The following factors have been identified: p300/ $\mathrm{CBP}^{26}$ acetylates p53 to increase p53 stability and activity; SOX4 is an important p53 regulator in the cellular response to DNA damage; ${ }^{6}$ MYBBP1A enhances the interaction between p53 and p300, which promotes p53 acetylation in response to ribosomal stress; ${ }^{27}$ and RPL11 is required for $p 53$ acetylation and p300 recruitment to the promoter regions of the p53 target gene in response to ribosomal stress. ${ }^{28}$ In this study, we found that NLK binds to not only p53 but also to MDM2, and NLK stabilizes p53 by abrogating the p53-MDM2 interaction. We also found that NLK inhibits MDM2-mediated ubiquitination of p53 and promotes p53 acetylation at Lys382, and this modification increases p53 activity by increasing the DNAbinding ability of $\mathrm{p} 53 .^{9}$

We show that NLK is induced in response to DNA damage in a p53-independent manner. The significance of this finding is that deletion of NLK reduces the stability and activation of p53. Because NLK interacts with p53 in physiological conditions, we hypothesize that NLK is required for maintaining a basal level of p53 expression. When DNA damage occurs, the NLK protein is rapidly induced to abrogate the p53-MDM2 interaction and promote p53 stabilization, and we describe this regulation mode in Figure 6. It is noteworthy that the mechanism by which NLK stabilizes p53 has nothing to do with its kinase activity. We supposed that NLK may regulate p53 function and activity in response to DNA damaged in a manner independent of its kinase function. a

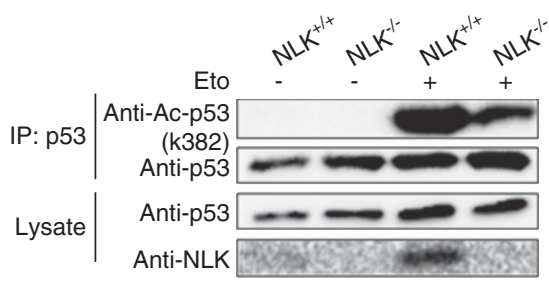

C

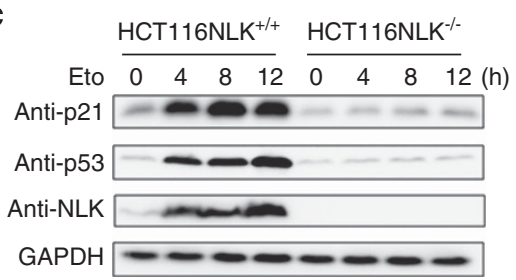

b

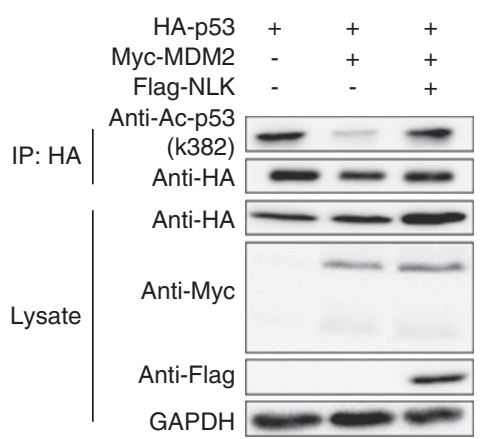

d
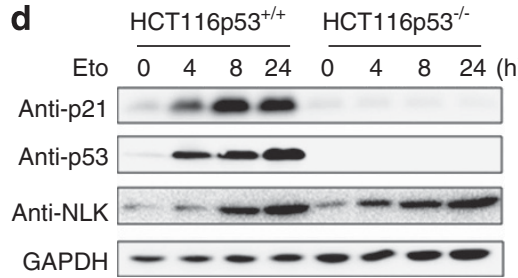

Figure 5 NLK affects p53 acetylation and downstream gene expression. (a) Acetylation of p53 at Lys382 decreases in the Eto-treated HCT116 NLK ${ }^{-1-}$ cells. HCT116 $\mathrm{NLK}^{+1+}$ and HCT116 NLK ${ }^{-1-}$ cells were treated with or without Eto, as indicated, for $12 \mathrm{~h}$; then, the cell lysates were immunoprecipitated with an anti-p53 (DO-1) antibody and analyzed by immunoblot analysis using the indicated antibodies. (b) NLK restores p53 acetylation at Lys382 in the presence of MDM2. HEK293 cells were transfected with $1 \mu \mathrm{g}$ of HA-p53, $1 \mu \mathrm{g}$ of Myc-MDM2 and $1 \mu \mathrm{g}$ of Flag-NLK expression vectors, as indicated. Forty-eight hours later, cell lysates were immunoprecipitated with an anti-HA antibody and analyzed by immunoblot analysis using the indicated antibodies. (c) NLK regulates p21 protein levels. HCT116 NLK ${ }^{+/+}$and HCT116 NLK ${ }^{-1-}$ cells were treated with $0.5 \mathrm{mM}$ Eto, and the cells were harvested at the indicated times. The cell lysates were analyzed by immunoblot analysis with the indicated antibodies. (d) HCT116

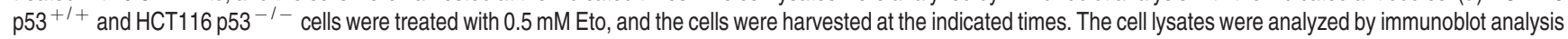
using the indicated antibodies 

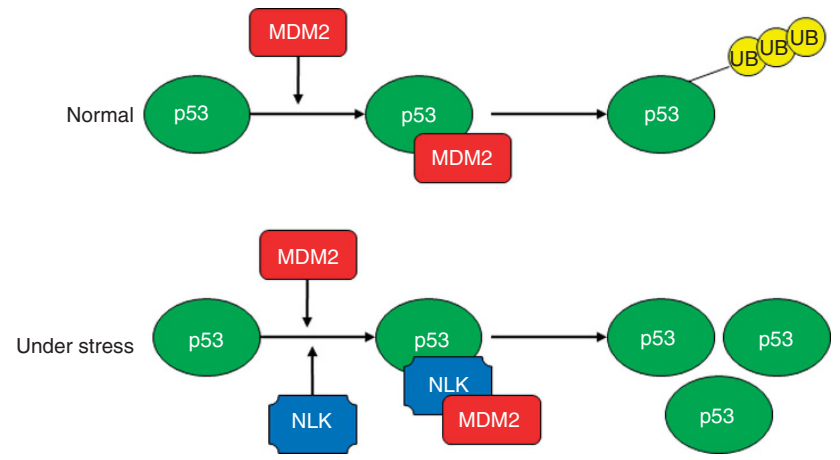

Figure 6 Model of NLK regulation of p53 stability. Under normal conditions, the p53 protein level remains low because of MDM2-mediated ubiquitination and degradation. Under DNA damage conditions, NLK abrogates the p53-MDM2 interaction and promotes p53 stabilization

It has been reported that NLK may function as a tumor suppressor by downregulating $\beta$-catenin/TCF complex transcriptional activity, which suppresses carcinoma cell growth. ${ }^{17}$ Clinical data show that NLK expression is significantly associated with the pathologic cancer grade and is correlated with cell proliferation and apoptosis. ${ }^{29,30}$ Recently, NLK has been associated with hepatocellular malignant transformation and proliferation. ${ }^{31}$ Thus, the precise role of NLK in tumorigenesis is still unclear and warrants further investigation.

The limitations of our study should be considered. The mechanism by which NLK abrogates the p53-MDM2 interaction requires further study. Another limitation of our study may be the pathway by which NLK affects p53 acetylation: the question persists of why NLK stabilization of p53 has nothing to do with its kinase activity. We will investigate these issues in future studies.

In conclusion, we have demonstrated that NLK is an important p53 regulator that responds to DNA damage. Furthermore, NLK interacts with p53 to enhance its stability and activity by abrogating MDM2-mediated p53 ubiquitination and degradation. Taken together, our findings suggest that NLK has an important role in tumorigenesis and may be a potential target for anticancer therapy in the future.

\section{Materials and Methods}

Cell culture. Human HCT116, NLK ${ }^{-1-}$ HCT116 and p53 ${ }^{-1-}$ HCT116 cells were cultured in McCoy's $5 \mathrm{~A}$ medium (Applichem, Darmstadt, Germany) supplemented with $10 \%$ fetal bovine serum (FBS, HyClone, Logan, UT, USA) and $100 \mathrm{U}$ of penicillin-streptomycin (Gibco, Carlsbad, CA, USA) at $37^{\circ} \mathrm{C}$ in a $5 \%$ $\mathrm{CO}_{2}$ incubator. HEK 293 cells were cultured in Dulbecco's modified Eagle's highglucose medium (HyClone) with $10 \% \mathrm{FBS}$ and penicillin-streptomycin in a $5 \% \mathrm{CO}_{2}$ incubator.

Antibodies and agents. An anti-rabbit monoclonal antibody against NLK (cat\# A400-046A) was purchased from Bethyl Laboratories, Inc. (Montgomery, TX, USA). An anti-mouse monoclonal antibody against p53 (DO-1) was purchased from Santa Cruz Biotechnology, Inc. (Dallas, TX, USA). Eto and an anti-mouse monoclonal antibody against anti-FLAG M2 were purchased from Sigma-Aldrich Co. LLC (St. Louis, MO, USA). An anti-mouse monoclonal anti-HA tag antibody (cat\# M132-3) was purchased from MBL, Inc. (Nagano, Japan). An anti-mouse monoclonal antibody against GAPDH (cat\# CW0266A) was purchased from Beijing Cowin Biotech Co., Ltd (Beijing, China). An anti-rabbit polyclonal antibody against acetyl-p53 (Lys382) was purchased from Cell Signaling Technology, Inc. (Beverly, MA, USA). An anti-mouse monoclonal anti-c-Myc antibody (cat\# 11667203001) was purchased from Roche Ltd (Indianapolis, IN, USA).
Glutathione Sepharose 4B (GST, cat\# 17075601) was purchased from GE Healthcare Bio-Sciences AB (Shanghai, China).

Plasmids. Plasmids expressing Flag-NLK and its mutations and HA-p53 and its mutations were generated using PCR and cloned into PCDNA5/FRT/TO-Flag/HA; Myc-MDM2 was generated using PCR and cloned into pCMV-3Tag-2A. GST-p53 was generated using PCR and cloned into pGEX 6p-1. Cherry-NLK was generated using PCR and cloned into pCherry-C3. GFP-p53 was generated using PCR and cloned into pEGFP-N1. Myc-ubiquitin was kindly provided by Hong-Bing Shu (Wuhan University, Wuhan, China). pGL3-TK-luc and pGL3-p53-luc were purchased from Addgene (Cambridge, MA, USA). GST-NLK was generated by PCR and cloned into pGEX 6p-1. His-p53 was generated by PCR and cloned into pET-28a $(+)$.

Transient and stable transfection. Transient transfections were performed with Lipofectamine 2000 (Invitrogen, Grand Island, NY, USA) according to the manufacturer's instructions (Life Technologies, Inc., Grand Island, NY, USA), as described previously. ${ }^{32}$ Transfection reagents and DNA were mixed in OptiMEM (Invitrogen); the complex was added to cells grown to $40-80 \%$ confluence, and the cells were cultured for approximately $4 \mathrm{~h}$, after which the medium was replaced with fresh medium. For stable transfection with pcDNA3.1 NLK-Flag, $1 \mathrm{mg} / \mathrm{ml} \mathrm{G} 418$ was added to the medium $48 \mathrm{~h}$ after transient transfection, and the cells were selected for 2 weeks. The stable cells were maintained in $0.5 \mathrm{mg} / \mathrm{ml}$ G418 medium.

Reporter assay. The reporter assay was performed as described previously. ${ }^{33,34}$ Cells $\left(2 \times 10^{5}\right)$ were transfected with $50 \mathrm{ng}$ of pGL3-p53-luc (for p53 activation), $10 \mathrm{ng}$ of $p G L 3$-TK-luc and the indicated levels of expression constructs using Lipofectamine 2000 according to the manufacturer's instructions. Luciferase activity was determined using the luciferase assay system and chemiluminescent reagents from Promega (Madison, WI, USA).

Immunoblot analysis. Immunoblot analysis was performed as described previously. ${ }^{32}$ Briefly, cells were cultured on $60-\mathrm{mm}$ dishes to $80 \%$ confluence and were then scraped and centrifuged at 1000 r.p.m. for $5 \mathrm{~min}$, followed by a wash in ice-cold PBS. Finally, the cells were lysed with SDS-sample buffer and boiled for $10 \mathrm{~min}$. The protein concentration was determined using a BCA protein assay kit (Thermo, Madison, WI, USA). Samples $(30 \mu \mathrm{g})$ were loaded onto SDS-PAGE. Proteins were transferred to polyvinylidene difluoride membranes (PVDF, Millipore, cat\# IPVH00010, Merck KgaA, Darmstadt, Germany). The membranes were blocked with TBST (PBS, $0.1 \%$ Tween 20 ) containing $5 \%$ dry fat milk and probed with primary antibodies overnight at $4{ }^{\circ} \mathrm{C}$, followed by incubation with an HRPconjugated secondary antibody (Jackson, West Baltimore Pike, West Grove, PA, USA) for $1 \mathrm{~h}$ at room temperature; the membranes were then developed using Immobilon Western Chemiluminescent HRP Substrate kits (Merck Millipore).

Immunostaining. Immunostaining was performed as described previously. ${ }^{35}$ HCT116 cells were cultured in 24-well plates with coverslips (cat\# 801007; NEST, Wuxi, China) and fixed with $4 \%$ paraformaldehyde (Electron Microscopy Sciences $16 \%$ Paraformaldehyde, Cat. 15700 , diluted into PBS) for $15 \mathrm{~min}$. Cells were then washed three times with PBS and permeabilized in $0.2 \%$ Triton X-100 in PBS for $5 \mathrm{~min}$. Cells were then washed with PBS and incubated with DAPI $(1 \mu \mathrm{g} / \mathrm{ml})$ for $15 \mathrm{~min}$. Immunostained cells were visualized and photographed using an Olympus Laser Scanning Confocal Microscope under a $\times 100$ oil objective.

Immunoprecipitation. Immunoprecipitation assay was performed as described previously. ${ }^{33,34}$ Cells were lysed in lysis buffer $(20 \mathrm{mM}$ Tris- $\mathrm{HCl}, \mathrm{pH}$ 7.4-7.5, $150 \mathrm{mM} \mathrm{NaCl}, 1 \mathrm{mM}$ EDTA, $1 \%$ NP-40, $10 \mu \mathrm{g} / \mathrm{ml}$ aprotinin, $10 \mu \mathrm{g} / \mathrm{ml}$ leupeptin and $1 \mathrm{mM}$ phenylmethylsulfonyl fluoride). Cell lysates were then incubated with the indicated antibody and Protein G-Agarose (Roche Ltd) at $4{ }^{\circ} \mathrm{C}$ for $2 \mathrm{~h}$. The Sepharose beads were washed three times with $1 \mathrm{ml}$ of lysis buffer containing $500 \mathrm{mM} \mathrm{NaCl}$. Precipitates were subjected to SDS-PAGE, and subsequent immunoblot analysis was performed using the indicated antibodies.

GST pull-down assay. The expression and purification of GST and His recombinant proteins were performed as described previously. ${ }^{35}$ In brief, GST and GST fusion proteins were expressed and purified according to the bio-protocol (Abcam, Cambridge, MA, USA). Flag-NLK proteins, obtained from the whole-cell lysates of HCT116 cells stably expressing Flag-NLK, were incubated with GST and GST-p53 fusion protein bound to Sepharose beads in $1 \mathrm{ml}$ of RIPA buffer 
(150 mM NaCl, 1.0\% NP-40, 0.5\% sodium deoxycholate, 0.1\% SDS, 1\% PMSF and $50 \mathrm{mM}$ Tris, $\mathrm{pH}$ 7.4.) at $4{ }^{\circ} \mathrm{C}$ for $4 \mathrm{~h}$. Beads were then washed and eluted in $50 \mu \mathrm{l}$ of $2 \times$ SDS/PAGE sample buffer and detected by immunoblot analysis.

His-p53 fusion proteins were expressed and purified according to the Molecular Cloning and were incubated with GST or the GST-NLK fusion protein bound to Sepharose beads in $1 \mathrm{ml}$ of RIPA buffer at $4{ }^{\circ} \mathrm{C}$ overnight. The beads were washed and eluted in $50 \mu \mathrm{l}$ of $2 \times$ SDS/PAGE sample buffer and detected by immunoblot analysis.

Lentiviral infection. HCT116 cells (CCTCC, Wuhan, China) were grown in McCoy's 5A supplemented with 10\% FBS (Invitrogen), penicillin (100 U/ml) and streptomycin $(100 \mu \mathrm{g} / \mathrm{ml})$ at $37^{\circ} \mathrm{C}$ in a $5 \% \mathrm{CO}_{2}$ incubator. The NLK-shRNA and control-shRNA lentiviruses were purchased from Cyagen Biosciences, Guangzhou, China. The stem sequence for the NLK-shRNA hairpin is $5^{\prime}$-CGGATAGACCTATT GGATATG- $3^{\prime}$. The stem sequence for the control-shRNA is $5^{\prime}$-GCGCGCTTTGTA GGATTCG-3'. This sequence has been widely used in other studies ${ }^{36}$ and does not match any known human coding cDNAs based on a blast search in GenBank. HCT116 cells were infected with the lentivirus, and the knockdown efficiency was confirmed by immunoblot analysis.

Clone formation. A colony formation assay was performed, as previously described, to detect the viability and tumorigenicity of cells..$^{32}$ In brief, cells $\left(2 \times 10^{3}\right)$ were seeded in six-well plates and were treated with or without Eto $(0$, $0.5,1$ or $2 \mu \mathrm{M}$ ) for $12 \mathrm{~h}$ the following day, after which the medium was replaced with fresh medium. After 10 days, clones were stained with crystal violet and photographed. Each experiment was repeated three times.

Cell proliferation assay. The cell proliferation assay was performed as described previously. ${ }^{32}$ In brief, cell proliferation was assessed using a Cell Counting Kit-8 (CCK-8; Dojindo Laboratories, Kumamoto, Japan) in accordance with the manufacturer's protocol. Cells $\left(1 \times 10^{4}\right)$ were seeded in a 96-well plate and treated with or without Eto $(0.5 \mu \mathrm{M})$ for $12 \mathrm{~h}$ on the following day, after which the medium was replaced with fresh medium. On the third day, the medium was replaced with $100 \mu \mathrm{l}$ of fresh McCoy's $5 \mathrm{~A}$ complete medium containing $10 \%$ CCK-8 reagent, and the cells were incubated for $1 \mathrm{~h}$. The absorbance was measured at $450 \mathrm{~nm}$ using a microplate reader (ELx800; BioTek, Winooski, VT, USA). Each experiment was repeated three times.

Statistics. All results are based on at least three independent experimental replicates. The statistical significances of the differences between the means were evaluated using Student's $t$-test performed as a paired, two-tailed distribution of arrays and are presented as $P$ values.

\section{Conflict of Interest}

The authors declare no conflict of interest.

Acknowledgements. This work was supported by grants from the National Basic Research Program of China (2011CB944404), the National Natural Science Foundation of China (81270306), the National Science and Technology Support Project (2012BAI39B02, 2012BAl39B03), the Trans-Century Training Programme Foundation for the Talents by the State Education Commission (NCET-10-0655) and Fundamental Research Funds for the Central Universities (204275771).

1. Bartek J, Bartkova J, Lukas J. DNA damage signalling guards against activated oncogenes and tumour progression. Oncogene 2007; 26: 7773-7779.

2. Bartkova J, Horejsi Z, Koed K, Kramer A, Tort F, Zieger $\mathrm{K}$ et al. DNA damage response as a candidate anti-cancer barrier in early human tumorigenesis. Nature 2005; 434 : 864-870.

3. Lane DP. Cancer. p53, guardian of the genome. Nature 1992; 358: 15-16.

4. Kastan MB. Wild-type p53: tumors can't stand it. Cell 2007; 128: 837-840.

5. Lu X. p53: a heavily dictated dictator of life and death. Curr Opin Genet Dev 2005; 15 : $27-33$.

6. Pan X, Zhao J, Zhang WN, Li HY, Mu R, Zhou T et al. Induction of SOX4 by DNA damage is critical for p53 stabilization and function. Proc Natl Acad Sci USA 2009; 106: 3788-3793.
7. Haupt $\mathrm{Y}$, Maya R, Kazaz A, Oren M. Mdm2 promotes the rapid degradation of p53. Nature 1997; 387: 296-299.

8. Kruse JP, Gu W. Modes of p53 regulation. Cell 2009; 137: 609-622.

9. Brooks CL, Gu W. Ubiquitination, phosphorylation and acetylation: the molecular basis for p53 regulation. Curr Opin Cell Biol 2003; 15: 164-171.

10. Liu N, Wang J, Wang J, Wang R, Liu Z, Yu Y et al. ING5 is a Tip60 cofactor that acetylates p53 in response to DNA damage. Cancer Res 2013; 73: 3749-3760.

11. Brott BK, Pinsky BA, Erikson RL. Nlk is a murine protein kinase related to Erk/MAP kinases and localized in the nucleus. Proc Natl Acad Sci USA 1998; 95: 963-968.

12. Thorpe CJ, Moon RT. Nemo-like kinase is an essential co-activator of Wnt signaling during early zebrafish development. Development 2004; 131: 2899-2909.

13. Ishitani T, Hirao T, Suzuki M, Isoda M, Ishitani S, Harigaya $\mathrm{K}$ et al. Nemo-like kinase suppresses Notch signalling by interfering with formation of the Notch active transcriptional complex. Nat Cell Biol 2010; 12: 278-285.

14. Ishitani T, Kishida S, Hyodo-Miura J, Ueno N, Yasuda J, Waterman M et al. The TAK1-NLK mitogen-activated protein kinase cascade functions in the $\mathrm{Wnt}-5 \mathrm{a} / \mathrm{Ca}(2+)$ pathway to antagonize Wnt/beta-catenin signaling. Mol Cell Biol 2003; 23: 131-139.

15. Yasuda J, Yokoo H, Yamada T, Kitabayashi I, Sekiya T, Ichikawa H. Nemo-like kinase suppresses a wide range of transcription factors, including nuclear factor-kappaB. Cancer Sci 2004; 95: 52-57.

16. Emami KH, Brown LG, Pitts TE, Sun X, Vessella RL, Corey E. Nemo-like kinase induces apoptosis and inhibits androgen receptor signaling in prostate cancer cells. Prostate 2009; 69: 1481-1492.

17. Yasuda J, Tsuchiya A, Yamada T, Sakamoto M, Sekiya T, Hirohashi S. Nemo-like kinase induces apoptosis in DLD-1 human colon cancer cells. Biochem Biophys Res Commun 2003; 308: 227-233.

18. Lord CJ, Ashworth A. The DNA damage response and cancer therapy. Nature 2012; 481: 287-294.

19. Pommier $\mathrm{Y}$, Leo $\mathrm{E}$, Zhang $\mathrm{H}$, Marchand $\mathrm{C}$. DNA topoisomerases and their poisoning by anticancer and antibacterial drugs. Chem Biol 2010; 17: 421-433.

20. Hande KR. Etoposide: four decades of development of a topoisomerase II inhibitor. Eur J Cancer 1998; 34: 1514-1521.

21. Porteus MH, Cathomen T, Weitzman MD, Baltimore D. Efficient gene targeting mediated by adeno-associated virus and DNA double-strand breaks. Mol Cell Biol 2003; 23: 3558-3565.

22. Liu Y, Li S, Zhang H, Wan Z, Zhang X, Du R. A one-step cloning method for the construction of somatic cell gene targeting vectors: application to production of human knockout cell lines. BMC Biotechnol 2012; 12: 71.

23. Sakaguchi K, Herrera JE, Saito S, Miki T, Bustin M, Vassilev A et al. DNA damage activates p53 through a phosphorylation-acetylation cascade. Genes Dev 1998; 12: 2831-2841.

24. Vousden KH, Lu X. Live or let die: the cell's response to p53. Nat Rev Cancer 2002; 2 : 594-604.

25. Harper JW, Elledge SJ. The DNA damage response: ten years after. Mol Cell 2007; 28: 739-745.

26. Wang X, Taplick J, Geva N, Oren M. Inhibition of p53 degradation by Mdm2 acetylation. FEBS Lett 2004; 561: 195-201.

27. Kuroda T, Murayama A, Katagiri N, Ohta YM, Fujita E, Masumoto $\mathrm{H}$ et al. RNA content in the nucleolus alters p53 acetylation via MYBBP1A. EMBO J 2011; 30: 1054-1066.

28. Mahata B, Sundqvist A, Xirodimas DP. Recruitment of RPL11 at promoter sites of p53regulated genes upon nucleolar stress through NEDD8 and in an Mdm2-dependent manner. Oncogene 2012; 31: 3060-3071.

29. Zhang Y, Peng C, Wu G, Wang Y, Liu R, Yang S et al. Expression of NLK and its potential effect in ovarian cancer chemotherapy. Int J Gynecol Cancer 2011; 21: 1380-1387.

30. Cui G, Li Z, Shao B, Zhao L, Zhou Y, Lu T et al. Clinical and biological significance of nemo-like kinase expression in glioma. J Clin Neurosci 2011; 18: 271-275.

31. Jung KH, Kim JK, Noh JH, Eun JW, Bae HJ, Xie HJ et al. Targeted disruption of Nemo-like kinase inhibits tumor cell growth by simultaneous suppression of cyclin D1 and CDK2 in human hepatocellular carcinoma. J Cell Biochem 2010; 110: 687-696.

32. Li SZ, Zhang HH, Zhang JN, Zhang ZY, Zhang XF, Zhang XD et al. ALLN hinders HCT116 tumor growth through Bax-dependent apoptosis. Biochem Biophys Res Commun 2013; 437: 325-330.

33. Zhong B, Yang Y, Li S, Wang YY, Li Y, Diao F et al. The adaptor protein MITA links virussensing receptors to IRF3 transcription factor activation. Immunity 2008; 29: 538-550.

34. Huang J, Liu T, Xu LG, Chen D, Zhai Z, Shu HB. SIKE is an IKK epsilon/TBK1-associated suppressor of TLR3- and virus-triggered IRF-3 activation pathways. EMBO J 2005; 24: 4018-4028.

35. Zhang $X$, Guo A, Yu J, Possemato A, Chen $Y$, Zheng $W$ et al. Identification of STAT3 as a substrate of receptor protein tyrosine phosphatase T. Proc Natl Acad Sci USA 2007; 104: 4060-4064.

36. Zhang L, Fogg DK, Waisman DM. RNA interference-mediated silencing of the S100A10 gene attenuates plasmin generation and invasiveness of Colo 222 colorectal cancer cells. J Biol Chem 2004; 279: 2053-2062. 